\title{
Cloud Services Platform based on Big Data Analytics and its Application in Livestock Management and Marketing
}

\author{
Jiayi Huang ${ }^{1}$ \\ Guangdong Institute of Modern Agricultural Equipment, Guangzhou, 510630, China \\ E-mail: huangjy@e-jiankun. com
}

\section{Peipei Guo ${ }^{2}$}

Guangzhou Jiankun Network Technology Development Co., Ltd., Guangzhou, 510630, China

E-mail: guoppee-jiankun.com

\section{Qiubo Xie}

Guangdong Institute of Modern Agricultural Equipment, Guangzhou, 510630, China

E-mail: xieqb@e-jiankun.com

\section{Xiangbao Meng}

Guangdong Institute of Modern Agricultural Equipment, Guangzhou, 510630, China

E-mail: mengxb@e-jiankun.com

To accelerate the development of the livestock industry, it is important to further enhance the spread of information, improve application ability of information technology, and expand the market for livestock products. According to the actual needs of modern livestock marketing management, this article combined e-commerce with traditional business model to build integrated cloud services platform of modern livestock marketing management, by using network technology, cloud computing technology, database technology and marketing management technology. For livestock enterprises, the platform can intelligently analyze information of livestock products supply and demand, while provide application services, including e-commerce, customer relationship management, inventory management, and agricultural products safety and traceability management, in order to realize online sales of livestock products and direct docking of livestock products with supermarkets. It is advantageous for livestock industry to promote the transformation of traditional to modern, improve management level, enhance the competitiveness, and promote the economic benefits.

ISCC 2015

18-19, December, 2015

Guangzhou, China

${ }^{1}$ This study is supported by Science and Technology Planning Project of Guangdong Province (2013B040400008) and Pearl River S\&T Nova Program of Guangzhou (2014J2200091).

${ }^{2}$ Corresponding Author: Jiayi Huang, Senior Engineer. 


\section{Introduction}

With the transformation of production model and adjustment of industrial structure, the construction of livestock information service support system is facing new challenges. Informationization is an important part of building a modern animal husbandry that is entering a new transition. To transform traditional animal husbandry with modern information technology is a significant and urgent task. Where there is no livestock informationization, there is no livestock modernization. Its informationization has been the trend of the development of the modern animal husbandry, and is the only way to sustainable development. In China, livestock information degree directly decides the depth and breadth of the livestock development.

With the new and increasing development of the economy of agriculture and rural areas, animal husbandry has gradually evolved into the predominant industry in rural areas[1]. However, some management problems of traditional animal husbandry have been highlighted, such as market information lag, scattered and small-scale enterprises, low efficiency [1-2]. So, the traditional mode can not meet the needs of current livestock informationization. With the rapid development of Internet technology and computer technology, a large number of livestock information sites emerged with a certain increasing rate to provide information on animal, veterinary, feed, technology and so on. However, it is not only difficult for users to choose information they needed from large amount of data, but also can not satisfy the demands of the users to buy and sell. Therefore, Guangzhou Jiankun Network Technology Development Co., Ltd., in the basis of the experience of two important livestock operators portals ("Guangdong pig information network" and "Guangdong veterinary information network") and the needs of livestock marketing, have created a modern livestock cloud services platform by using cloud computing technology and big data analytics, which combines e-commerce with information services and traditional business model as one set[3-7]. The platform can provide a wide range of application services for customers, such as e-commerce, customer relationship management, inventory management, to enhance the level of e-commerce application of our province.

\section{Technical Architecture of Platform}

The platform in the structures is divided into three levels, including IaaS (Infrastructure as a Services), PaaS (Platform as a Services) and SaaS (Software as a Services)[8-9], as shown in Figure 1.

\subsection{IaaS Layer}

IaaS offers a variety of end-user-oriented services, including computing, storage, networks and other fundamental computing resources. On this basis, the operational service providers can deploy and run arbitrary software, such as operating system and applications. End users don't have to manage or control any cloud computing infrastructure, but can use the corresponding resources at anytime and anywhere, and must be restricted by safe network components (eg. firewall, load balancer, etc.) to ensure data security. 


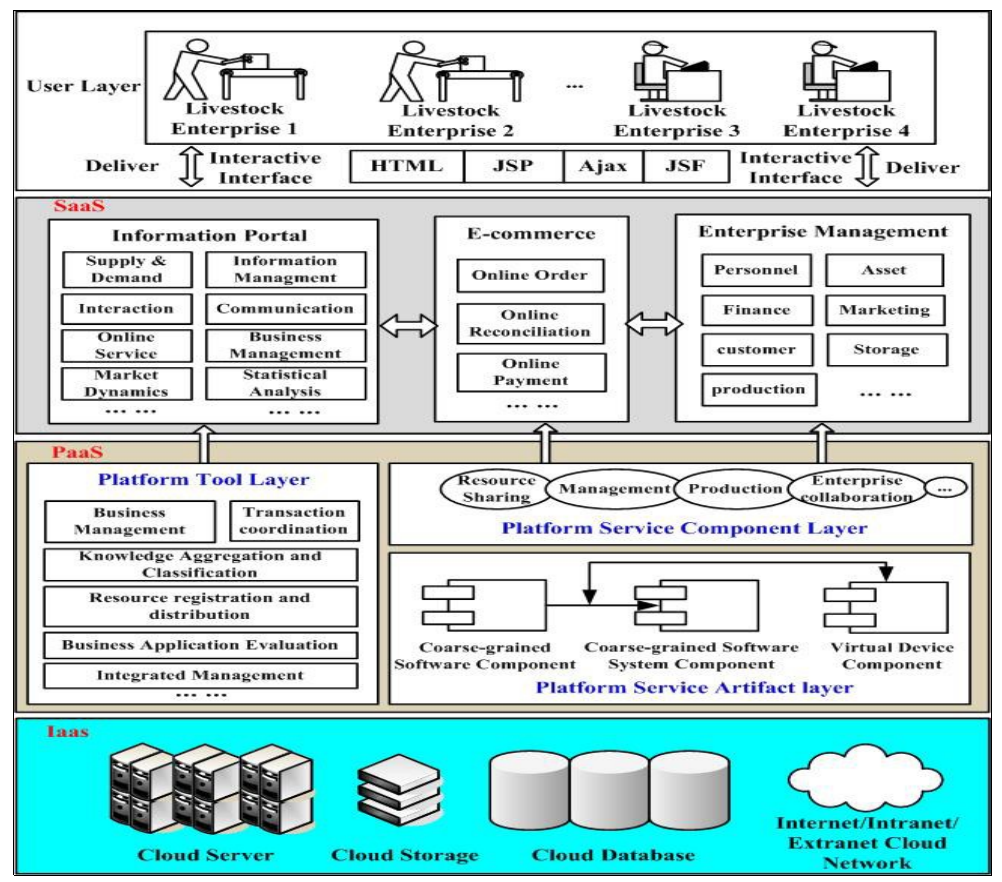

Figure 1: Architecture Diagram of Cloud Services Platform on Modern Livestock Marketing Management.

\subsection{PaaS Layer}

PaaS provides a component-based architecture platform for supporting platform configurability. The component-based architecture is a decomposition of a large application system into hundreds of small business processes, and each business process can be combined by components. When business needs change, a new combination of components can quickly and easily change business process or implement new business. In addition, the application of component technology will also save development costs and shorten the development cycle, improve the reusability of knowledge and the stability of the system. PaaS layer consists of three parts:(1) Platfotm service component layer: store and manage all kinds of coarse-grained service components with different granularity rang for various service calls. (2) Platform service artifact layer: form different business service components for various business model calls. (3) Platform tool layer: offer a series of tool set to support registration, publish, search matching, calls and management of various types of components.

\subsection{SaaS Layer}

The end-user-oriented services providing by SaaS is an application that runs on IaaS and is composed of service components and artifacts. Users can access SaaS on general devices through the client interface, such as a browser, without the need to manage or control any cloud computing infrastructure, including network, servers, storage, etc. SaaS has two layers, business model layer and user layer. The former is a user-oriented application, including information portal website, e-commerce and application services. The latter is responsible for all management interfaces connecting end-users, as an important window for users to use a variety of cloud resource services.

\section{The Main Function of the Platform}


In this paper, the cloud services platform of modern livestock marketing management mainly includes two aspects: one is the combination of e-commerce means with the traditional business model to comprehensively promote high quality animal husbandry products from large-scale farms or professional farmers through the Internet. In order to achieve online sales of animal products, "online store" with local specialty products was established and other new emerging channels were carried out. Based on the integrated system of data mining, intelligent prediction and visualization display, the intelligent analysis system of agricultural supply and demand information was formed, by which the spatial distribution of major agricultural products, trends of market price, and intelligent docking of supply and demand information were shown to provide e-commerce services for livestock farmers and even ordinary backyard farmers. Through demonstration and application, the online sales of animal products could improve livestock operation and management level.

The other is the construction of the platform to provide application outsourcing services for livestock enterprises, including e-commerce, customer relationship management, inventory management, etc. The establishment of livestock quality and safety traceability system helps to guide the improving quality of animal products, promote livestock products directly into a supermarket, and then achieve the direct docking of agricultural products and supermarkets.

\section{Key Technology}

\subsection{Security of Enterprise Data}

For cloud platform users, the biggest worry is data security, that is data loss or damage to other people. This system adopts specific solutions: (1) By using the architecture of singleinstance and multi-tenant, the mode of shared database and independent user can ensure logical isolation of customer data and relative independent of each other. Currently, most of the platform vendors selected this solution. (2) By multi-tenant data model, it is easy to provide users with database self-management solutions. (3) A variety of classification defense mechanism through trusted links, encrypted data, authentication and authorization were used to provide customers with the security services. (4) Users must combined USBKEY with the account number and password to login system, to enhance security login. (5) Make detailed logs record all users' information, such as name, login time, login IP, etc., and providing statistical reports. (6) Allow users to set their own account to use period of time, out of which the account is not allowed to log in or operate, letting users have more confidence. (7) Separate data server and application software server to ensure the security of enterprise data. (8) System automatic backup data in the local daily and offsite regularly, to make sure data security. (9) Sign and make security commitments to increase the confidence of users.

\subsection{Big Data Storage and Concurrent Access}

One of the important subjects for management information system based on cloud platform is solving the problem of data storage and concurrent access under the condition of large-volume user application. In view of mass storage problem, we have taken the following solutions: (1) Select high-performance servers and ORACLE system to increase the processing capacity of massive data. (2) Make software architecture support distributed applications to achieve load balancing. (3) Establish backup and recovery mechanisms of dynamic data to classify dynamic business data by period. (4) Isolate query data and operational data in logic, to 
ensure the query speed of system. (5) Optimize query and statistical algorithms of various large amount of computation. (6) Make data be scalable to maximize data reading speed and its storage capacity.

For large concurrent user access problem, we have offered the following responses: (1) Use multiple database support cloud platform, and carry forward the database schema. As a server cluster infrastructure, application middleware adopts load balancing mode. (2) Select AJAX technology to realize client asynchronous request. (3) Optimize user queue scheduling to reduce "traffic jam" phenomenon of user access. (4) Take "optimistic locking", a kind of more relaxed locking mechanism based on data version recording mechanism, concurrency control user actions. (5) Set a time to the millisecond field in database table and read this field during program processing. (6) Oversee the operations of the system through quantifying the SLA (Service Level Agreement) operating standard, to ensure a high availability of application system. (7) Enable application scalability to maximize parallelism.

\subsection{Platform Differentiation Control}

How to meet customers' demands for differentiation and personalization is the key issue for sustainable application of management software and services platform. For resolving the above issue, we use the following solution: metadata is used to provide users with the customization and configuration of applications to meet their specific needs. As a result, users can change configuration of the four fields in two levels (metadata and semantic layer). Four specific fields are: (1) User interface and brand: The platform provides relevant features to users to customize interface, forms, buttons and links, as well as relevant contents that can change graphics, colors, fonts, and so on. (2) Workflow and business rules: Users can configure the customized workflows with a variety of online software provided by the platform to fit their own business process requirements. (3) Scalable data model: It enables users to freely make applications according to their needs change tables, fields, etc.. (4) Access control: Security policy tacks each user's permission, and the user can configure the security policy.

\section{Technology Realization}

Considering the modern livestock cloud services platform needs to face a lot of customers, the platform has the configurability, scalability, multi-tenant efficiency and ease of use. All these determine the technology architecture of the system design. Therefore, we design the following system architecture, as shown in Figure 2.

\subsection{Directory Services}

Directory services are for users to quickly query server resource information in basis of directory database, by which all the information and resources of the directory distributed in different servers can play a role, so as to achieve adaptive of users and application servers, and improve speed of multiple concurrent query.

\subsection{Load Balancing Server and Access Control}

Load balancing server and access control are mainly to solve the problem of resource (hardware and software resources) sharing and coordination between multiple application servers under certain control rights. On the load balancing server group, the platform provides 
hosting services for different customers by running a same instance. Each application instance serves a number of customers with different needs that is managed by the load balancing server group made as a unified interface to interact with users. Same instance can maximize the sharing of resources among users, but will not let the end user aware applications are shared with multiple users. Different customer data are separated from each other, while every customer only can access to data within their respective purview through authorization and security strategies, to distinguish different customer data.

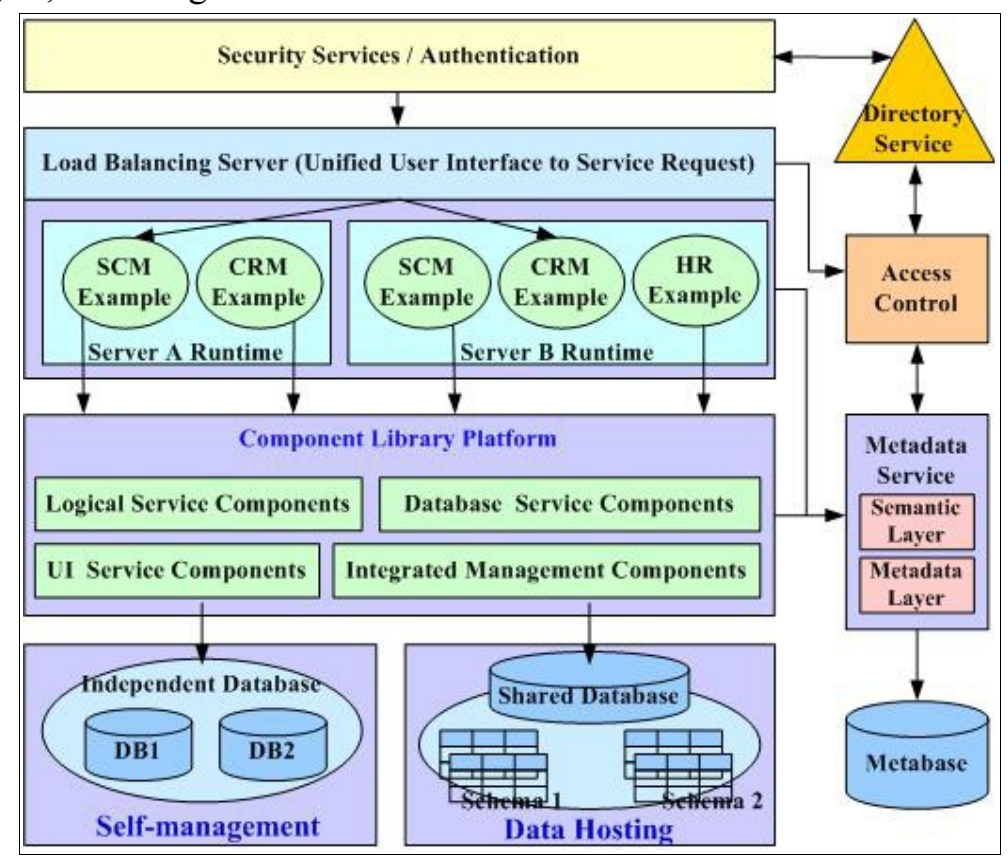

Figure 2: System Structure Diagram of Cloud Services Platform on Modern Livestock Marketing Management

\subsection{Metadata Services and database}

Metadata services and its database include two layers, semantic layer and metadata layer, providing customization and configuration application for customers to meet their specific needs. Through the metadata layer stored in the metabase, customers can modify, extend, or even re-define data table structure, views, field, type, etc. Through the semantic layer, customers can reconfigure user interface and customize report format and content. Through metadata services, customers can adjust user interface, set workflow and business rules, extend data model, control access, etc.

\subsection{Component Library Platform}

Component library platform is one of the main means to ensure the scalability of the application system. Component of the SOA architecture decides the degree of the platform configuration. Component library platform has four types of components: (1) Logical service components, including models, application frameworks, system architectures and design patterns of an enterprise information system domain, are comprehensive reflections of the application model. (2) UI service components, including user interface, print interface and other types of components, are composed of several view components used to realize user interfaces. (3) Database service components, include database engine components, workflow engine components, and various middleware service components, etc. (4) Integrated management 
components, a group of standardized parameters providing interfaces to integrate third-party systems to meet the system scalability.

\subsection{Database System}

The system gives two kinds of customer data storage environment for enterprises to choose: a shared database environment and independent database environment. The former is a data hosting environment. Customer data are stored in a shared database server of the platform, provides function services, such as data import, export, backup, restore, isolate, transfer, etc. The latter is a customer self-management environment. Customer data are stored in a separate database server. Customers self-manage their own databases.

In shared database environment, an independent database schema is selected. That is, although different customers use a same database, each of them has their own set of tables to form their special mode. This method provides a logical data isolation for the users requiring a high security, but cannot achieve complete isolation system, which is applicable to the applications with a relatively small number of the forms in database. It can reduce the cost of operations, but customers must accept that their data with others' data sharing a same database.

In independent database environment, a centralized self-management is used to provide customers with their own dedicated database. To realize the centralized management, individual data keep connection with platform centralized data and consistent with the specific data from the centralized data. It is relatively simple to recover the users' data from backup in the event of a failure. Therefore, it is more suitable for the customers who are willing to pay a higher price for a greater security and customizability customers.

\section{Analytics}

In this paper, the model that combines e-commerce means with the traditional business, catches a lot of customers' concerns, which come from all over the country with a variety of different occupations. The number from the Guangdong province accounts for more than one third, while the proportion of Guangxi, Fujian, Shandong, Hunan, Henan province and other provinces as well(Figure 3). Meanwhile, this platform promotes high quality animal husbandry products from enterprises, farms or professional farmers with the help of Web server and pageviews(Figure 4).

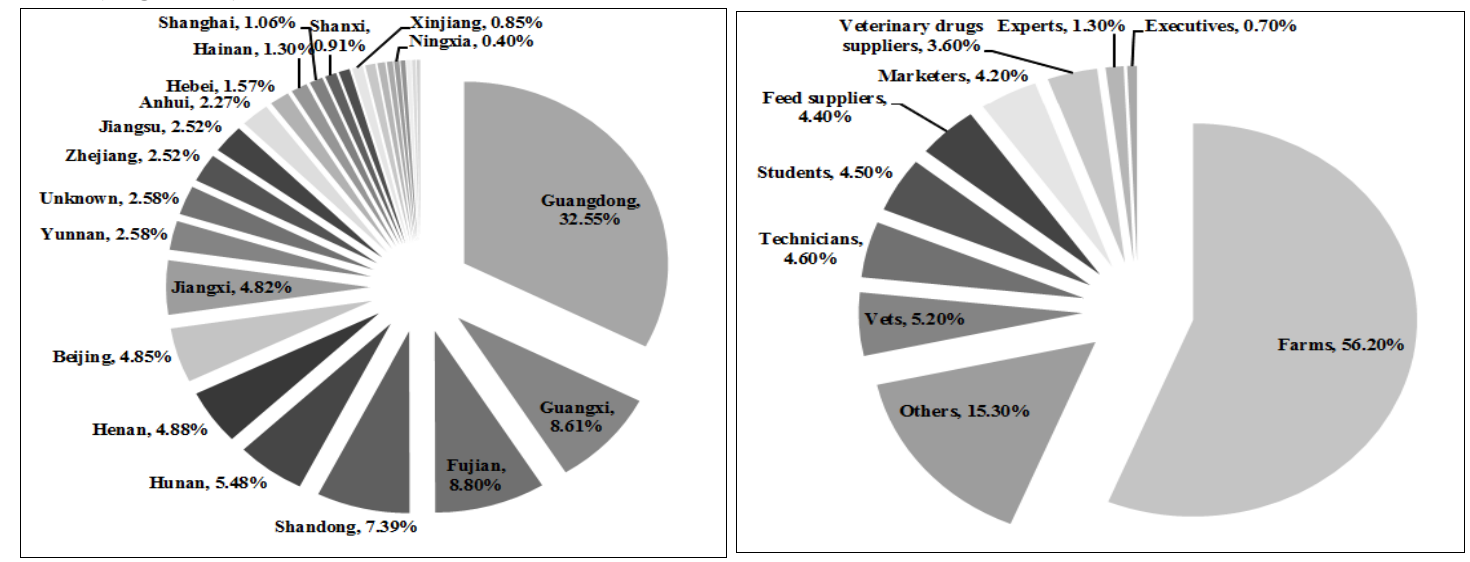

Figure 3: Geographical and Occupational Analysis of the Users 


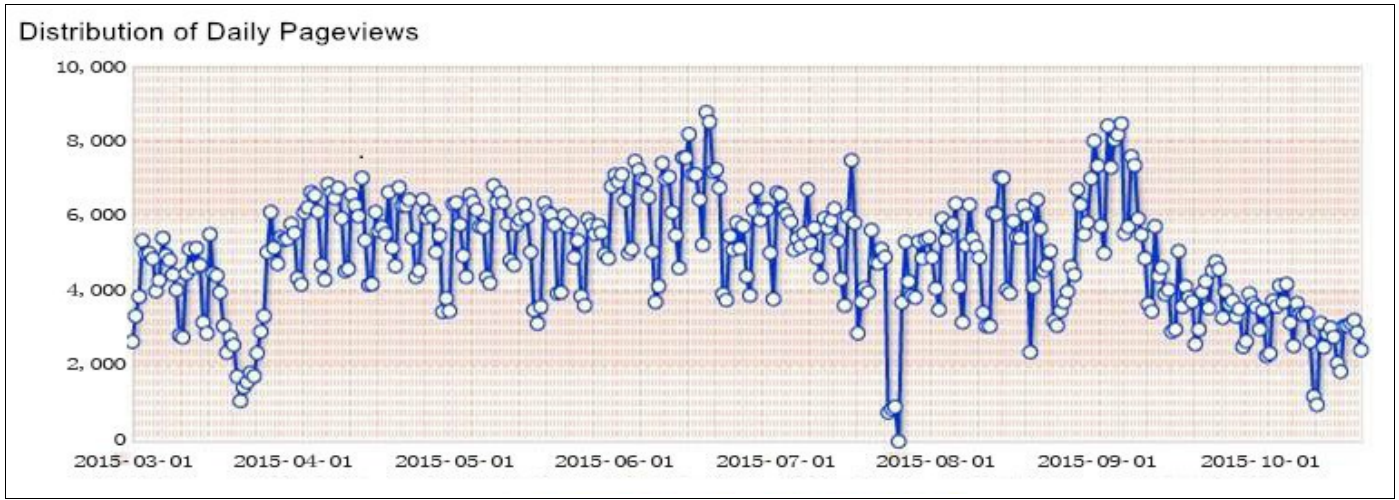

Figure 4: Distribution of Daily Pageviews from the platform

\section{Conclusion}

The emergence of cloud computing meet the information needs of the small livestock enterprises. It effectively reduces the initial investment of the enterprises and satisfies the changing needs of the users, while it makes small and medium-sized enterprises enjoy the advantages of the technology and adapt to the business needs of remote office. The platform uses the Internet to provide outsourcing services for livestock enterprises, such as e-commerce, customer relationship management, inventory management, etc., and helps livestock enterprises selling products and improving production management level by the means of combining ecommerce and traditional business model. In the current, such a business model is a brand new model, having great vitality and development prospects.

\section{References}

[1] J. Han, X. Wang, A Network-Based Management Information System for Animal Husbandry in farms[J]. IFTP AICT. 317: 49-54(2010).

[2] X. Zhang, S. Y. Xiang, Analysis and Design of Animal Husbandry E-Commerce Platform Based on UML and MVC[J]. Service Science and Management. 4(4B): 97-103(2015). (In Chinese)

[3] J. Z. Luo, J. H. Jin, A. B. Song, F. Dong. Cloud computing: system architecture and key technologies[J]. Journal on Communications. 32(7): 3-21(2011). (In Chinese)

[4] P. Liu. Cloud computing[M]. Beijing: Publishing House of Electronics Industry. 9-12(2011). (In Chinese)

[5] J. Y. Wu, L. D. Ping, X. Z. Pan, Z. Li. Cloud computing from concept to platform [J].

Telecommunications Science. (12): 23-25(2009). (In Chinese)

[6] C. L. Liu . The integration of e-commerce and ERP based on SaaS model[D]. Chongqing:

Chongqing University(2007). (In Chinese)

[7] W. H. Wang. Research of e-commerce management model based on cloud computing[D]. Dalian: Liaoning Normal University(2012). (In Chinese)

[8] Z. Lin, R. F. Zhou, Y. C.Yang. Research of cloud computing job scheduling platform based on Web[J]. Science and Technology Information. (5): 156-157(2012). (In Chinese)

[9] P. Wang. Key technologies and application examples of cloud computing[M]. Beijing: Posts and Telecom Press. 73-90(2010). (In Chinese) 